\title{
Quality Control Testing of BCG Vaccine: Current Practices and Technological Advancements
}

\author{
Gaurav Pratap Singh Jadaun*, Harit Kasana and Neeraj Malik \\ National Institute of Biologicals (Ministry of Health and Family Welfare, Government of India), Institutional Area, Noida, India
}

Received: January 03, 2016; Accepted: January 13, 2016; Published: January 24, 2016

*Corresponding author: Gaurav Pratap Singh Jadaun, National Institute of Biologicals (Ministry of Health and Family Welfare, Government of India), A-32, Sector-62, Institutional Area, Noida, India, 201309, Tel: +91-0120-2400022; Fax: +91-0120-2403014; E-mail: gpsj@rediffmail.com

\begin{abstract}
The Bacille Calmette-Guérin (BCG) is live and attenuated strain of Mycobacterium bovis which is used throughout the world as vaccine against tuberculosis. BCG vaccination contributes to the prevention and control of tuberculosis in limited situations when other strategies are inadequate. As there is currently no alternative, BCG will continue to be used in future as vaccine and also as a prime vaccine in a primeboost immunization in conjunction with new tuberculosis vaccines. Therefore, there is need to improve the characterization and the assays used for the quality control of BCG vaccine. In this article, we present the recent technological advancements in the quality control testing of BCG vaccine. Special attention has been given to the newly developed methods for the identification and potency testing of BCG taking into account the recent advancements in genetics and molecular biology.
\end{abstract}

Keywords: BCG, Quality Control; Multiplex PCR; ATP Bioluminescence; Colorimetric Assays; Flow Cytometry

\section{Abbreviations}

TB, tuberculosis; BCG, Bacille Calmette-Guérin; ZN, Ziehl Neelsen; PCR, polymerase chain reaction; CFU, colony forming unit; LJ, Lowenstein Jensen; MIRUs, mycobacterial interspersed repeat units; SNPs, single nucleotide polymorphisms; VNTR, variable number tandem repeats; WHO, World Health Organization

\section{History of BCG Development}

TB is caused by Mycobacterium tuberculosis and is among the leading cause of human deaths from an infectious disease. A vaccine named BCG is available against TB for human use since 1920s which shows protection against childhood TB and tuberculous meningitis [1]. The BCG vaccine was discovered by two French scientists namely Albert Calmette, a physician and Camille Guérin, a veterinarian at Pasteur Institute, Lille, France. The BCG vaccine contains a live and attenuated strain of $M$. bovis that was originally isolated from a cow with tuberculous mastitis. The isolate was subcultured on glycerinated potato bile medium every 3 weeks for a period of 13 years during 1908-
1921 and a total of 231 passages. This long process resulted in loss of virulence of the bacillus and changes in phenotypic characteristics. The live, attenuated BCG strain was first given orally to infants in Paris in 1921. Afterwards, new methods for BCG vaccination were introduced including intradermal, multiple puncture and scarification. BCG is also used as an effective cancer immunotherapy [2] and developed as a recombinant vehicle for multivalent vaccines against other diseases [3].

The original strain of $M$. bovis used to prepare BCG was maintained by serial passage at the Pasteur Institute, until it was lost or discarded. Before its loss, it was distributed to different laboratories in many countries that maintained their own daughter strains through passaging. Serial subculturing on various culture media under different conditions maintained by different laboratories has resulted in the production of many heterogeneous daughter strains (substrains) that differed widely in colony morphology, growth characteristics, biochemical activity and their efficacy to provide protection against TB.

\section{Quality Control of BCG Vaccine}

Quality control testing of BCG vaccine is performed by manufacturers and control laboratories in compliance with the pharmacopoeial requirements to ensure the quality, safety and efficacy [4]. The current methods for BCG vaccine testing have their own limitations as they were developed many years ago. A number of unresolved issues in the quality assessment of BCG vaccines have been identified. These include genetic differences of substrains and their implications to phenotypic characteristics, immunological mechanisms of efficacy and protection induced by different BCG vaccines, immunogenicity and efficacy in animal models and the impact of vaccine characteristics on the safety profile of vaccine preparations. In addition, recently reported findings on antimicrobial resistance of some strains raised an issue of their relevance in the quality assessment of BCG vaccines [5].

\section{Molecular Advancements in Identification of BCG}

The current method employed for identification of BCG is ZN 
staining together with colony characteristics on solid medium [4] The ZN staining method is inadequate because of inherent nonspecificity of the procedure. Moreover, the slow growth of BCG on the solid medium makes its identification delayed upto four weeks. BCG is genetically and phenotypically similar to M. bovis and to other species of the M. tuberculosis complex. Therefore, biochemical properties and growth characteristics used for identification of $M$. bovis BCG have their own limitations and are not definitive. Also, these methods provide only limited substrain differentiation. The current pharmacopoeial monograph for freeze-dried BCG vaccine states that molecular biological techniques may be used as alternatives for identification [4].

Subtractive genomic hybridization had identified genetic differences between virulent strains of $M$. bovis and $M$. tuberculosis and avirulent BCG [6]. Among the genetic differences identified, the RD1 region is shown to be absent in BCG strains and present in M. bovis and M. tuberculosis complex strains [7]. Based on these findings the RD1 region is targeted to develop multiplex PCR system to distinguish different BCG substrains and differentiate BCG from virulent mycobacterial strains $[7,8]$. This method has also been evaluated to assess its accuracy, robustness and reproducibility for use as an identity test for BCG vaccine [8]. In another study, multiplex PCR assay has demonstrated itself to be rapid, specific, reproducible and robust and is proposed as a novel test for BCG identification [9].

\section{Viable Count for Potency of BCG}

Assay of potency plays a central role in the quality control of BCG vaccine and ensures that the successive vaccine lots are consistent and within predefined specifications. The current method for determination of the number of viable cells in a BCG vaccine depends on the counting of CFU produced after culture on solid medium [4]. LJ medium is the culture medium of choice for this test. Although viable count per se is not an assay of potency; however, it is used as a surrogate marker for BCG potency. Determining the content of viable cells by CFU in production lots of freeze-dried BCG vaccine has disadvantages in relation to the length of time required to obtain results and lack of reproducibility of the assays due to aggregation of cells. Hence manufacturers and control laboratories are looking for a rapid and reproducible potency assay for BCG. Various methods have been proposed as alternative of CFU for the BCG vaccine including assays based on bioluminescense, colorimetry and flow cytometry.

\section{ATP Bioluminescence Assay}

As an alternative to the viable count method, abioluminescense assay has been developed and may be used for potency testing of BCG provided that the method is properly validated against the culturable particle test. ATP bioluminescence assay for viability testing of the BCG vaccine is developed and validated at Statens Serum Institute, Denmark [10]. Authors used lyophilized BCG vaccine samples from Danish collection and stored for various periods at different temperatures to obtain variations in viability. Intracellular ATP was then extracted using boiling tris-EDTA buffer and the ATP concentration was measured in presence of luciferin/luciferase. Simultaneously, the number of CFU per vial was determined by plating of serial dilutions and the results of two methods were compared. The ATP bioluminescence method was shown to be robust and applicable to determine ATP content in lyophilised BCG for estimating viability in the BCG samples with high correlation with the viable count [10]. The ATP assay is more rapid and provides good reproducibility but has disadvantage in quantitatively extracting total ATP and the subsequent stability of the extracts. In addition, this technique like conventional viable count is adversely affected by bacterial cell clumping.

\section{Colorimetric Assay}

Colorimetric assays make use of the tetrazolium salts which have been used extensively in determining the viability of cells including bacteria. In colorimetric assays tetrazolium salts are metabolically reduced to highly colored end products called formazans, the quantity of formazan produced being directly proportional to viable cell count. Colorimetric assays using oxidation-reduction dyes have also developed for determining minimum inhibitory concentrations of antibiotics for pathogenic mycobacteria [11]. These assays are simple, inexpensive and give rapid results in comparison with conventional methods. Using the same principle, Kairo et al. have used two tetrazolium salts, namely XTT and MTT, to develop a colorimetric method for determining number of viable BCG organisms [12]. This assay provides more rapid and reproducible results in comparison with the conventional viable count. XTT showed greater sensitivity than MTT with lower detection limit of about $7 \times 10^{4}$ $\mathrm{CFU} / \mathrm{ml}$. Assay results show good correlation with CFU; however, disagreements were observed for the BCG lots which were on the borderline of acceptability for viable counts. Colorimetric assay takes 48 hours to give final results as compared with 4 weeks for conventional method. One major concern associated with the use of colorimetric assays is the generation of aerosol resulting in safety concerns [11].

\section{Flow Cytometry Assay}

Flow cytometry is a very sensitive, efficient assay and does not require any cell culturing or enrichment procedures. These assays utilize fluorescent dyes as sensitive indicators to detect and distinguish the cell viability in many cell types. A flow cytometric assay has been developed to measure the viable counts of $M$. bovis BCG and to compare with the conventional viable count method [13]. In the assay propidium iodide is generally used to label dead cells. To evaluate the reliability of the developed flow cytometric assay, the viable counts obtained for BCG vaccine samples from the flow cytometric method were compared to the conventional viable counts on LJ medium. The total cell numbers in BCG preparations were measured by Coulter counter Z1 directly or counted by the Flow-Count Fluorespheres during the flow cytometric assay. The results showed that the total cell numbers measured by the Coulter counter Z1 directly were more accurate. This newly developed assay needs only 4 hours to be fully completed as compared to 4 weeks by conventional method. The results of the flow cytometry assay are 
all read out from instruments, which reduces the variation from different operators to overcome the limitation of conventional CFU method

\section{Protective Potency Assay}

In terms of quality control of BCG, a key issue is lack of correlates of protection and therefore the absence of a tool to distinguish 'protective' from 'non-protective' vaccines in the laboratory. Various animal models (such as mouse, guinea pig) have been used to develop assays for quantitative measurement of protective efficacy of BCG vaccines [14]. Among these models, different challenge routes (respiratory, intra-tracheal, intravenous, subcutaneous, intra-peritoneal) and different challenge strains (H37Rv, Erdman, Beijing) are in use. Protective potency assay of guinea pig model has been used for seed lot testing of BCG vaccine in Japan [15]. However, there is a need for standardization of these assays before their use in routine testing of BCG vaccines.

\section{In vitro Approaches in Testing Virulence}

Virulence of the BCG is tested to ensure the safety of vaccine preparation and guinea pigs are used for routine monitoring of the presence of virulent mycobacteria in BCG vaccine [4]. This assay is very time consuming as the animals are under observation over six weeks after injection of BCG vaccine. As an alternative, a PCR-based in vitro assay has been developed which differentiates BCG strains and virulent mycobacteria. This PCR procedure targets the intergenic region separating two genes encoding a recently identified mycobacterial two-component system, named SenX3-RegX3 [16]. The senX3-regX3 intergenic region contains a novel type of repetitive sequence, called MIRUs. All tested BCG strains exclusively contained 77-bp MIRUs within the senX3-regX3 intergenic region, whereas all nonBCG $M$. tuberculosis complex strains contained a 53-bp MIRU, in addition to the 77-bp MIRUs. Application of this method in future will reduce the time required for testing absence of virulent mycobacteria in BCG products. Also, replacement of animal assay with PCR based method will solve the ethical considerations.

\section{Characterization of BCG in Post Genomic Era}

Availability of genomic and proteomic tools to study molecular genetics of the mycobacteria has paved the way for rigorous analysis of genomic characterization as well as to understand the protective immunity of different BCG strains. The complete genome sequence were determined for various BCG strains in recent past $[17,18,19]$. Comparative genomic tools have been used to study various daughter strains of BCG and regions of difference such as deletions, insertions and SNPs have been identified $[20,21]$. One such region named RD1 region has been shown to be associated with BCG attenuation [22]. Based on their genomic characterization, BCG vaccines were divided into the early strains, represented by BCGs Japan, Sweden, and Russia and the late strains, including BCGs Pasteur, Danish, Glaxo, and Prague [20]. Each substrain has its own signature genetic profile and molecular methods are described in the literature for characterization of BCG substrains. Genetic typing of BCG strains based on VNTR at MIRU loci has been suggested by WHO [23] and can be used for monitoring the consistency of BCG lot production.

Studies have reported that the estimates of the protection against TB imparted by BCG strains varied widely and several factors, such as genetic differences in the BCG strains used for immunization, environmental influences and host genetic factors contribute to this protection variability [1]. Using the proteomic approaches it is possible to identify the variations in protein profiles of different vaccine strains. Identification of differences in protein expression offers a way to decipher the genetic differences and their expression in the final vaccine preparation. Proteomic studies have shown that BCG strains with similar genomic content exhibit phenotypic differences that can be of significant importance for pathogenesis, immune response and variable efficacy of BCG vaccine [24]. Orduna and colleagues have used 2-D gel electrophoresis, protein sequencing and blotting methods to study the protein profile of BCG vaccine [25]. These proteomic approaches may also be utilized to identify any changes in protein profiles from working seed, different passage levels to final lot preparation of the BCG product. The exploration of the proteome of M. Bovis BCG strains provides valuable information regarding specific proteins, many of which have been implicated in protective immune responses, and helps defining the candidates for future vaccination strategies.

\section{Conclusions}

It is clear from the above description that quality control testing of BCG vaccine using current methods is time consuming and shows variations in results indicating the need for improvements in methods. Technological advancements, particularly in the field of genomics and proteomics, have enriched our understanding towards BCG and also led to the development of new methods for BCG testing to ensure the quality, safety and efficacy of BCG vaccine preparations. These methods provide rapid and consistent results and may replace the classical methods after proper validation.

\section{Acknowledgements}

We are thankful to Dr. Surinder Singh, Director, National Institute of Biologicals, Noida for his constant support and encouragement.

\section{References}

1. Fine P. Variation in protection by BCG: implications of and for heterologous immunity. The Lancet. 1995;346(8986):1339-1345.

2. Brosman SA. Bacillus Calmette-Guerin immunotherapy. Techniques and results. Urol Clin North Am. 1992;19(3):557-564.

3. Lugosi L. Theoretical and methodological aspects of BCG vaccine from the discovery of Calmette and Guerin to molecular biology. A review. Tubercle Lung Dis. 1992;73(5):252-261.

4. BCG vaccine, freeze dried. European Pharmacopoeia 8.0. Volume I. p. 819-820.

5. Hong DN, Huyen MN, Nguyen Thi Ngoc Lan, Duong NH, Ngo VV, Ngoc DT, et al. Rifampin-resistant Mycobacterium bovis BCG-induced disease in HIV-infected infant, Vietnam. Emerg Infect Dis. 2013;19(7):1168. doi: $10.3201 /$ eid1907.130025 
6. Mahairas GG, Sabo PJ, Hickey MJ, Singh DC, Stover CK. Molecular analysis of genetic differences between Mycobacterium bovis BCG and virulent M. bovis. J Bacteriol. 1996;178(5): 1274-1282.

7. Talbot EA, Williams DL, Frothingham R. PCR identification of Mycobacterium bovis BCG. J Clin Microbiol. 1997;35(3):566-569.

8. Bedwell J, Kairo SK, Behr MA, Bygraves JA. Identification of substrains of BCG vaccine using multiplex PCR. Vaccine. 2001;19(15):2146-2151.

9. Kasana H, Pathaniya L, Suchitra, Sikarwar G, Malik N. Quality evaluation of BCG vaccine by conventional and molecular methods. Int J Curr Res. 2015;7(9):20691-20692.

10.Jensen SE, Hubrechts P, Klein BM, Haslov KR. Development and validation of an ATP method for rapid estimation of viable units in lyophilised BCG Danish 1331 vaccine. Biologicals. 2008;36(5):308314. doi: 10.1016/j.biologicals.2008.05.001. Epub 2008 Jul 9.

11. Jadaun GPS, Agarwal C, Sharma H, Ahmed Z, Upadhyay P, Faujdar J, et al. Determination of ethambutol MICs for Mycobacterium tuberculosis and $M$. avium isolates by resazurin microtitre assay. J Antimicrob Chemother. 2007;60(1):152-155.

12. Kairo SK, Bedwell J, Tyler PC, Carter A, Corbel MJ. Development of a tetrazolium salt assay for rapid determination of viability of BCG vaccines. Vaccine. 1999;17(19):2423-2428.

13. Yang YC, Tsai MH, Cheng HF. Determine the potency of BCG vaccines by flow cytometer. Biotechnol Biotechnol Eq. 2011;25(2):2394-2398.

14. Castillo-Rodal AI, Castanon-Arreola M, Hernandez-Pando R, Calva JJ, Sada-Diaz E, Lopez-Vidal Y. Mycobacterium bovis BCG sub strains confers different protection against Mycobacterium tuberculosis infection in BALB/c model of progressive pulmonary tuberculosis. Infect Immun. 2006;74(3):1718-1724

15. Yamamoto T, Phalen S, Uchida K, Umemori K, Nojima Y, Horiuchi Y, et al. Protective efficacy of BCG Tokyo 172 in the guinea pig model of pulmonary tuberculosis. Kekkaku. 2000;75(5): 379-388.

16. Magdalena J, Supply P, Locht C. Specific differentiation between Mycobacterium bovis BCG and virulent strains of the Mycobacterium tuberculosis complex. J Clin Microbiol. 1998;36(9): 2471-2476.
17. Brosch R, Gordon SV, Garnier T, Eiglmeier K, Frigui W, Valenti P, et al. Genome plasticity of BCG and impact on vaccine efficacy. Proc Natl Acad Sci USA. 2007;104(13):5596-5601.

18. Seki M, Honda I, Fujita I, Yano I, Yamamoto S, Koyama A. Whole genome sequence analysis of Mycobacterium bovis bacillus CalmetteGuérin (BCG) Tokyo 172: a comparative study of BCG vaccine substrains. Vaccine. 2009;27(11):1710-1716. doi: 10.1016/j vaccine.2009.01.034. Epub 2009 Feb 4.

19. Gomes LH, Otto TD, Vasconcellos EA, Ferrao PM, Maia RM, Moreira AS, et al. Genome sequence of Mycobacterium bovis BCG Moreau, the Brazilian vaccine strain against tuberculosis. J.Bacteriol. 2011;193(19):5600-5601. doi: 10.1128/JB.05827-11.

20. Behr MA, Wilson MA, Gill WP, Salamon H, Schoolnik GK, Rane S, et al. Comparative genomics of BCG vaccines by whole genome DNA microarray. Science. 1999;284(5419):1520-1523.

21. Pelayo MCG, Uplekar S, Keniry A, Lopez PM, Garnier T, Garcia JN, et al A Comprehensive survey of single nucleotide polymorphisms (SNPs) across Mycobacterium bovis strains and M. bovis BCG vaccine strains refines the genealogy and defines a minimal set of SNPs that separate virulent $M$. bovis strains and $M$. bovis BCG strains. Infect Immun. 2009;77(5):2230-2238. doi: 10.1128/IAI.01099-08. Epub 2009 Mar 16.

22. Liu J TV, Leung AS, Alexander DC, Zhu B. BCG vaccines: their mechanisms of attenuation and impact on safety and protective efficacy. Hum Vaccine. 2009;5(2):70-78.

23. World Health Organization. WHO discussion on the improvement of the quality control of BCG vaccines. 2005.

24. Rodriguez-Alvarez M, Mendoza-Hernandez G, Encarnacion S, Calva JJ, Lopez-Vidal Y. Phenotypic differences between BCG vaccines at the proteome level. Tuberculosis (Edinb) 2009;89(2):126-135. doi: 10.1016/j.tube.2008.12.001. Epub 2009 Feb 20.

25. Orduna P, Cevallos MA, de Leon SP, Arvizu A, Hernandez-Gonzalez IL, Mendoza-Hernandez G, et al. Genomic and proteomic analyses of Mycobacterium bovis BCG Mexico 1931 reveal a diverse immunogenic repertoire against tuberculosis infection. BMC Genomics 2011;12:493. doi: 10.1186/1471-2164-12-493. 